Sireijen veridhafft bat. श्uf biejen Gsegenftand und feine Begründung

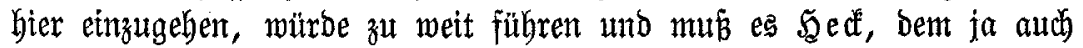
Das Şartig'idhe Merfabren unbefannt war, überlaffen bleiben mit ber

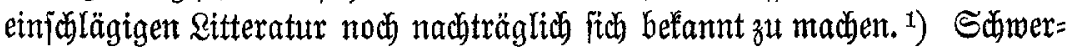
verftändlich wie bie Interitellung, Daß behufe einbeitlider 2lufarbeitung

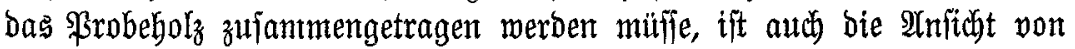

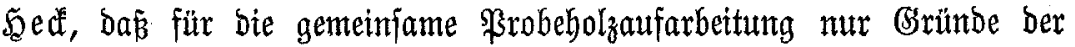
Bequentidyteit fich geltend machen lieken. Saandelt es fich ond in

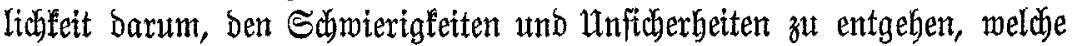

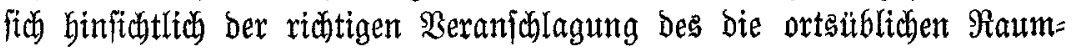
maßje mur teilmeife ausfülllenden \$robeholzes ergeben und bie bei $g e=$ trennter 2 uffarbeitung von beijpielsweije 20 Brobejtänmen fith auch 20 mal wieberbolen, wäbrent ite bei gemeinjamer stufbereitung nur $1 \mathrm{mal}$ in bie Erjoheinnng treten.

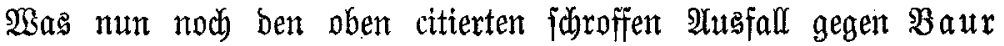
anbelangt, jo bätte berjelbe unt fo mebr unterbleiben müffen, als $\mathfrak{S} e d$

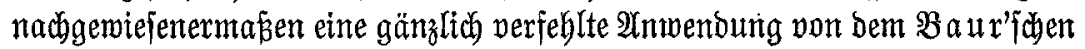
$M=K . H . \mathfrak{f}$. gemadht hat.

Darmftad, Nowember 1899.

\title{
Ein Walopflug.
}

Bon Forftmeifter $O$ fter held, Langenterg. ${ }^{2}$ )

Dex von Jahr zu Jahr fith ftetgernbe Mrbeitermangel madbt fich nach und nach jelbit in ben entlegeniten $\mathfrak{B a l}$ agebieten füblbar unb brängt ben Forftwint gleid bem \&anowirt zur Bermendung von Majojinen und

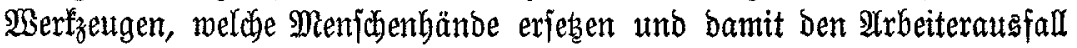
nach $\mathfrak{M}$ ioglichleit becten.

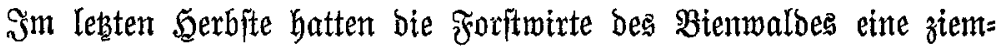
lich reidhe Eichenmaft und eine gute $B$ utdijen= Sprengmaft auszumüben.

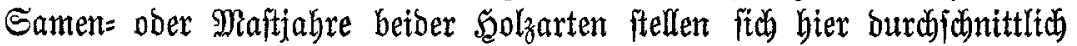
alle 5-7 Sabre ein und merben beshalb auth weniger ftarfe Samen=

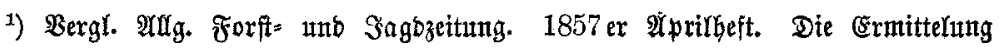

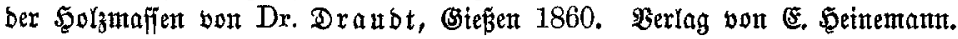

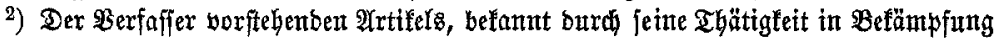
ber Sofiutte, ift leiber am 3. Uuguft 1899 verftorbent. 
erträge - Sprengmaiten - jorgfältig zu vermenben gejuctit. Dabei

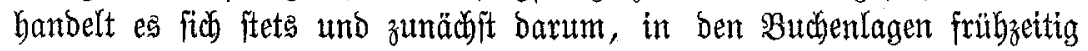

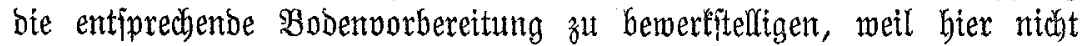

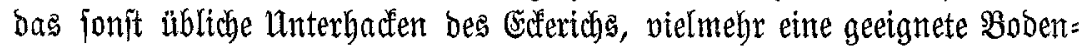
vermunbung vor Deffen 2 tbfall ben beiten Erfolg geliefert hat. Es murbe biaber befonberer Erfolg mit Furchenzieben erreicht und murben bieje Furden je nach ben Bobenverbältniffen in 0,50 bis $1 \mathrm{~m}$ breiten 2 (tb= ftänoen etwa $0,20 \mathrm{~m}$ tief mögliduft parallel hergeftellt. Sicht mintor guten Exfolg lieferte übrigens bas Sautenfoflagen, $\delta$. $\mathfrak{t}$. in etwa 0,25 bie $0,30 \mathrm{~m}$ breiten Abftänden wirb eine flache etwa 15 bis $20 \mathrm{~cm}$ tiefe Raute her= geftellt, weldhe bas Edderid gleidy ben Furdhen aufnimmt. Somobl Furdben

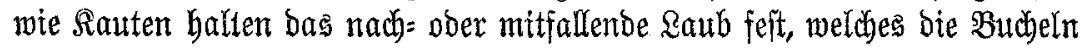

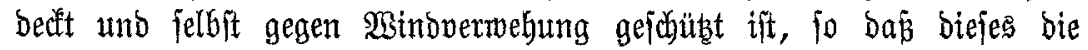
SBobenfrifche erbält und ben Sämling in gefäbrlicten Froftperioben ober bet intenfituer Iroctente nidbt jelten wohlthätig fibübt.

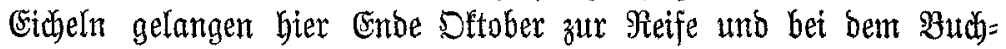
ecterig beginnt ber Mfball fojon um bie Mitte bes September. UIm oaher

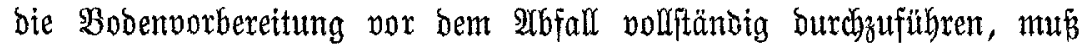
ichon anfangs 2 luguft bamit begomen merben. Nur jebr fajmer ift aber

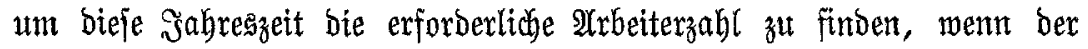
Forftwirt ohnehin mur mehr auf ben Rletnbauer angemiejen uno biejer

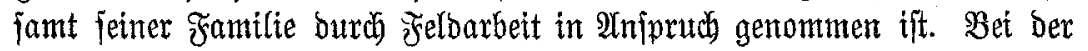
Budbmaft tim Sabre 1893 madbte fich ber Atrbeiterausfall allerbings audi fionn wabrnebmbar, allein immerbin waren nod weiblidbe 2 lrbettaträfte genügeno zu finbent, mit benten, fo gut es eben gint, bie ßobenvorberettung burchgeführt merben fonnte. Sim lesten Serbfte war bies jeboch wejent= lich anders gemorben.

Das Seftar biejer Bobenworbereitungen erforbert einen 2 Uufwand

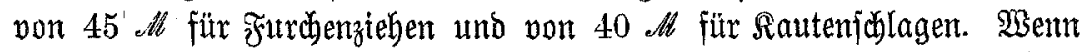
bies immerhin ein ziemlich hoher Roftenbetrag ift, jo war bei ber lekt= jäbrigen Maft boch meniger bie Roftenfrage als ber geidjilberte 2 Urbeiter= mangel Seranlaffung, bei ben bier vollfommen ebenent Terrainverbältniffert trib bem ziemlich lockeren uno tiefgründigen Diluvialboben nadi einem \$fluge zu forfthen, mit weldjem mittelft Pferbe= ober Ddjen=(Befpannes bas Furchenziteben bewerfitelligt werben tönne. Fin gejofidter Sdjmied fonitruierte uns benn audé ben im Modell bergeftellten Walopflutg, bei weldem bie Form bes Schares ben Schwerpunft bildete und funftio=

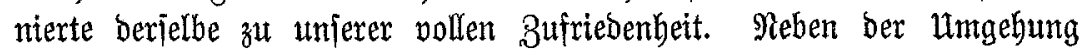

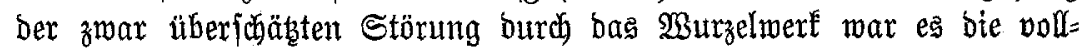

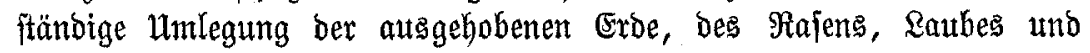


anderer Beitantsteile ber Bobenbecfe und bamit bie volfitänoige Dffen= legung ber Furche, was mit ber Form der Geftaltung bes \$fflugichares erreibst werden mupte. Wie aber bies im vollen unifang gelungen ift, möge auts folgenden Beifpielen hervorgehen:

Bunädaft murbe natürlich bie Bobennorbereitung für bie Buchen= bejamung in ber $\mathfrak{A}$ rt getroffen, baß in $0,50 \mathrm{~m}$ breitem arbitande Furchen

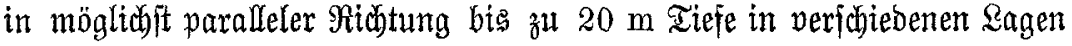
und in grofer 2 thtsbehmung gezogen wurben. Die 2 rebeit bot mider Er= warten feine Sdjwierigfeiten; ein Mann führte die \$Fferde, ein zweiter beherrichte ben \$flug. Bei ftärferen Mutzeln wurbe lebterer entiprectend

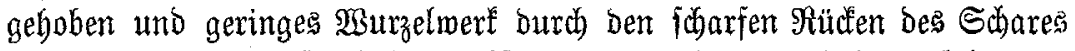

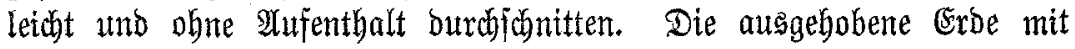

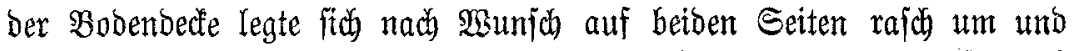

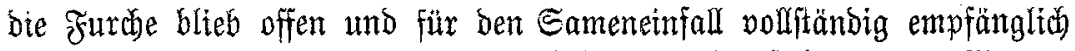
bergeftellt. Bei ber überall burdigeführten Schlagftellung bes Mutter= bejtandes boten aud bie vorbanbenent säume und Stöbie wentg

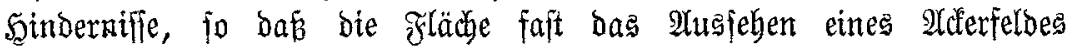
getwann.

Der Erfolg war benn aud überrajctend nno weit gröéper als in früherent Sabren, wo - wie oben erwähnt - Furchen mit ber Saand ge= zogen worben fino. Bei bem âflaufen ber Befamung erwedten wieber=

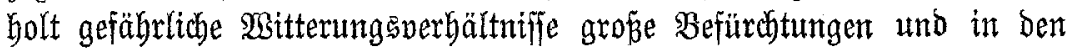

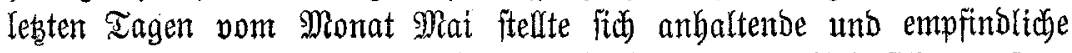
Irodfene ein. Whein bie reichlidje \$uthenbejamnng erbielt fich ungeftört und bürfte jeţt - Ento Sulti - um fo mebr gefidert jein, als bie meifen Sämlinge einen itarfen sohannitrieb entmičelt haben.

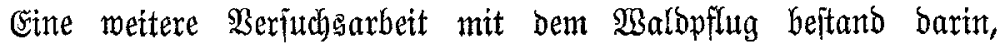
baß an etnem geeigneten Orte gletdhe Furdhen wie vorber gezogen uno in sieje bebufs Grünoung eines Eibjenhorites fofort Eicheln eingejäet

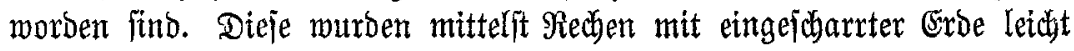

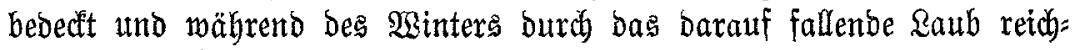

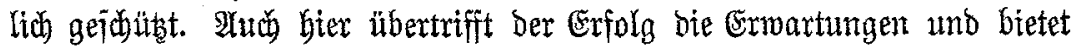
bie bejamung einen jebr befriebigenden 2 (nblič.

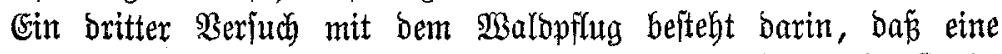

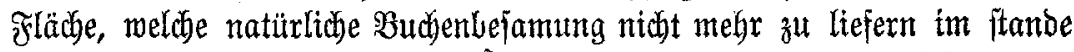
ift, wie in ben obigen F̧ällen gefurdst unb mit vierjäbrigen, nicht gerabe fchönen Buctenpflanzen bepflanzt worben ift. Die Pffanzung gefdhah in bie Frurchen auf $0,40 \mathrm{~m}$ Entfernung mittelfit Rlemmeifens vor bem $\mathfrak{a} \mathfrak{a} b=$

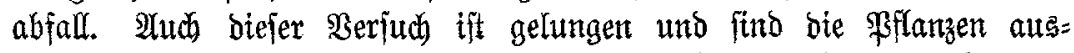
nabmslos frijch und bie Gejamtpflanzung vollfommen befriebigend. 
Bulest wurbe eine Fläbe mit Buthenvorbereitungsitellung ebenfalls veriuchsmeije beshalb gepflitgt, weil bamit nachgemiejen merben wollte,

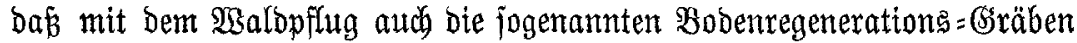
Gergeftellt werben founen. Dieje äureerit erfolgreide tndo allgemein empfehlensmerte $\mathfrak{U r b e t}$ tommt bod wohl in ben meiften Füllen beshalb

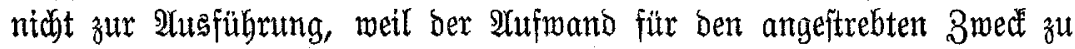

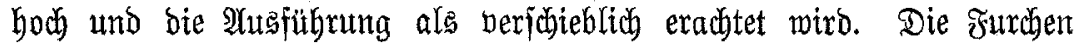
haben auch hter bas \&aub retalich aufgenommen, halten biejes jamt ber Bobenfrifde fejt und wurbe nebenbei ber $\mathfrak{B}_{0}$ ben foweit geloctert, daf beffen Thätigfeit wieber angeregt, bie Frobulftivität frijch eingeleitet unt für bie nachjolgende $\mathfrak{B}$ eriüngung des Beftandes wefentlich gefördert miro.

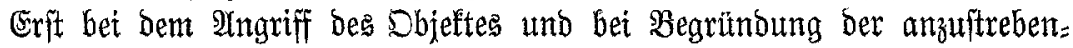
ben natürlithen Befamung wiro ber eminente Erfolg ber vorattggegantgenten

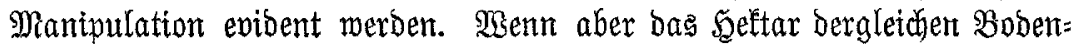
bearbeitung mit $15 \mathscr{M}$ bewerffitelligh merben fann, Darf vorausftedtlid

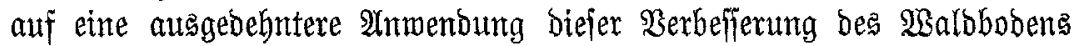
geredhnet werben als bies bei 40 und 50 Mro seftar bisher ber Fall war.

Wir tönnen ben Seerren Sollegen unt Factgenoffen nux ernitlich zu

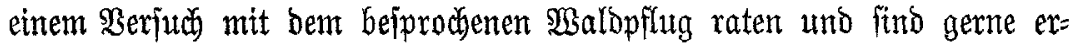
bötig, lesteren in Beitellung zu nehmen. Der $\mathfrak{F r e i s}$ miro fich fertig vom Sajmied auf 55 all belaufen.

\section{Über einige neue forftthädliche Tipulidenarten.}

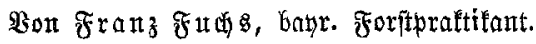

Die grö̈en langflügeltgen uno langbeinigen Schnaden, Sdfnauzen= müffen genannt, meldje man zu ben ripuliben im weiteren Sinne rechnet, befiben \&arwen, weldhe bem \&and= und Forftwirt bisweilen (d)äblich werben. Die Şmagines ftrto jebr bäuftige Titere, bie man bejonders auf tauigen Wiejen in ber Morgenfühle oft in grojer Bahl finden fann, aber

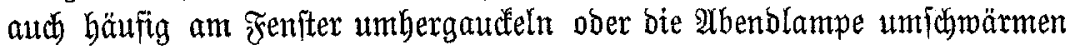
fieht und bie von lnnfundigen oft als ftechenbe Snjetten gefürubtet werden, bie aber ganz harmloje Gseidgöpie find, bie weder zu beisen noch zu ftechen

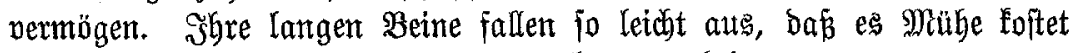
ein Exemplar unverlest in bie Samminng zu bringen.

Die Sarven leben teils im $\mathfrak{B}$ boen, wo man fie oft in grofer $3 \mathfrak{a}$ hl finden fann, anbere in Dünger, Sdhlamm ober moberndem $\mathfrak{S}_{2}$ lze. Shgre 